\title{
Further Observations on the Motility of Proteus vulgaris Grown on Penicillin Agar
}

\author{
By A. FLEMING \\ The Wright-Fleming Institute of Microbiology, St Mary's Hospital, \\ Medical School, London, W. 2
}

SUMMARY: The movement of the enormously enlarged filaments of Proteus vulgaris grown on penicillin agar ceased, or was slowed down, by screening the culture from the radiant heat of a microscope lamp. Organisms stimulated by heat after resting a short time in this way moved more rapidly than before. The increased activity was maintained for longer periods with longer rests, the increment in the period of activity increasing gradually as the resting period increased. In the condition of the test, rests of 60-120 sec. produced a maximal response.

Repeated heat stimulation rapidly exhausts the capacity of the organisms to maintain a steady rate of movement. Under constant stimulation organisms maintain a fairly constant rate for some hours and then slow considerably as though they or their flagella were exhausted. A small decrease in radiant heat energy can induce a - reversal in the direction of movement.

Active flagella attached to immobile organisms react to heat stimuli in the same way.

The effect of penicillin on the morphology of Proteus vulgaris has often been described. With concentrations between 3 and 10 units (u.)/ml. the filamentous forms of the microbe become very long and many of them become very distorted.

In a previous article I and my co-workers (Fleming, Voureka, Kramer \& Hughes, 1950) made observations on the motility of Pr. vulgaris grown on penicillin agar and examined by phase-contrast microscopy. We showed that when radiant heat from the microscope lamp was cut out by the insertion of a heat filter, the motility of the greatly enlarged filaments of Pr. vulgaris ceased or slowed down, and that the time during which an organism could maintain its maximum activity depended on the period of rest which preceded such movement. These findings applied also to flagella belonging to filaments which were themselves motionless. The present paper records an extension of these observations.

Technique. The organisms were grown in slide cultures as previously described. Cultures on agar containing $5 \mathrm{u}$./ml. of penicillin were incubated at room temperature. I was especially interested in the long filaments which were coiled like a watch-spring and whose only movement was a simple rotation. These could be watched for hours in the same field of the microscope. A sure way of obtaining these forms was to plant Pr. vulgaris in a thick streak across the cover-slip, and after this had dried to put two or three drops of penicillin agar at $50^{\circ}$ on the cover-slip and immediately invert it on a slide. Along the edges of the streak there were always many 'watch-springs' to be found.

The heat filter was that supplied by Kodak for their 'Retina' lantern; it absorbed only about $10 \%$ of the light but about $80 \%$ of the radiant heat. 
The flagellar movements or the rotations of the coiled filaments were timed by an assistant who noted to the nearest second the intervals called by the observer at the microscope. A better way was to use a wire recorder; the observer at the microscope talks into the microphone and then the record is played back and timed. This has the advantage that the times could be checked if there was any doubt.

Movement of the roatch-spring forms. The rate at which the coils rotate varied enormously with different cultures and with different organisms in the same culture. The rotations may be rapid-less than 1 sec.-or they may be very slow, up to 30 sec. or more.

In the older slide cultures almost all the organisms are immobile and only the 'watch-springs' continue their rotary movements. It seems strange that this should be so except that possibly in their rotary movement these organisms have worn a groove in the agar so that they are actually moving in a thin layer of fluid. Thus, they would have little resistance to overcome and so would be able to continue movement for a longer period than the others which, in their enlargement, became caught between the agar and the cover-slip.

The effect of $a$ heat filter. The effect of decreasing the radiant heat varies somewhat with different cultures and different organisms. Occasionally it has little effect on the rate of rotation of the 'watch-springs', but usually the organism either stops or slows down very considerably. The cessation of movement may be only temporary, and after a period varying from a few seconds to some minutes the rotation recommences slowly and eventually reaches about the same rate as it would had there been no heat filter for a considerable time. The effect on the visible flagella is the same. They usually stop completely and then start moving slowly.

The flagella are extraordinarily sensitive to the variation of the radiations caused by the interposition of the heat filter. The mere passage of the filter across the path of light so that it is effective for not more than one-fifth of a second is often sufficient to stop or slow down the movement of the flagella. The introduction of a heat filter may also induce a reversal of the directional movement of many filaments.

Effect of removing the heat filter. After a short latent period there is a violent movement of the flagella and the rate of rotation is markedly increased. The rotation is maintained at the increased rate for a variable time and then decreases (Table 1). Rotation usually slows to below its regular speed, as if the organism were exhausted by the violent effort and then gradually picks up again to its normal speed, which may be maintained for a long time.

Effect of inserting the heat filter for varying times. Fleming et al. (1950) showed that the number of revolutions which the watch-spring forms make at increased speed depends on the time during which the radiant heat is excluded. A very much larger series of observations has amply confirmed this relationship. Table 1 shows the results obtained with a single organism, stimulated to increased movement by exposure to radiant heat after varying periods without it.

When the time of rest is plotted against the number of revolutions before 
slowing, a regular curve is obtained (Fig. 1). There is a rapid rise in the number of revolutions following increases in the period of rest up to 20 or $30 \mathrm{sec}$. and then the curve flattens out and there is little difference between a rest period of 1 and 2 min.

Table 1. Rate of movement of a 'watch-spring' filament of Proteus vulgaris Period of
screening
radiant h
(sec.)
180
2
75
10
5
1
75 after varying periods of rest
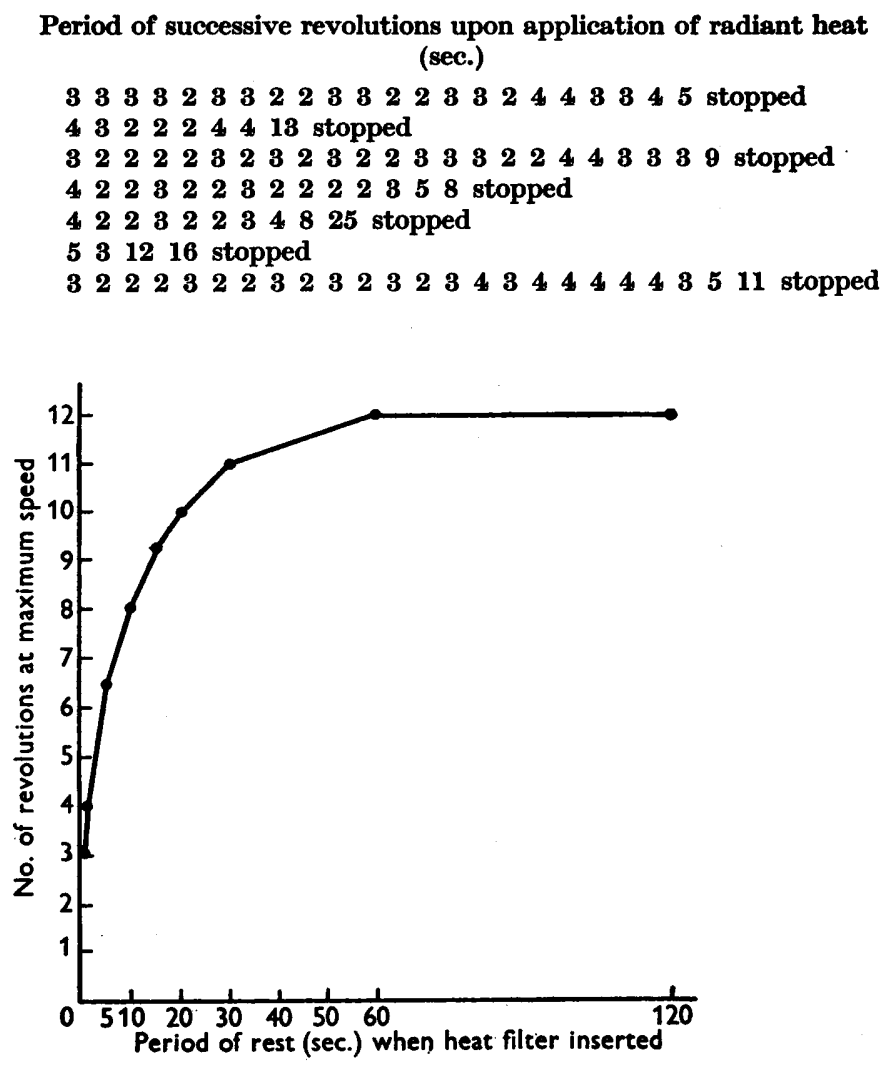

Fig. 1

The effect of repeated heat stimulation. In the experiment recorded in Table 1 there was apparently no exhaustion after repeated stimulation. It is usual, however, especially in older cultures, to see, after intermittent heat stimulation, that the organism becomes gradually more sluggish. In one experiment (Table 2) the time taken for each revolution increased, with the heat filter in, from $2.8 \mathrm{sec}$. to $14 \mathrm{sec}$, and with the heat filter out from 1.6 to 9 sec.

Period during which an organism can maintain its activity. A rotating watchspring form in a slide culture which had grown overnight at room temperature was timed at intervals for $5 \mathrm{hr}$., during which time it was exposed to radiant heat from the microscope lamp. The period of each of ten consecutive revolu- 
Table 2. Exhaustion of an organism after repeated heat stimulation to increased activity

Position of heat filter

In, at beginning of observation Out after $5 \mathrm{~min}$.

In after $2 \mathrm{~min}$.

Out after $4 \mathrm{~min}$.

In after 47 sec.

Out after $48 \mathrm{sec}$.

In after 25 sec.

Out after 36 sec.

In after 38 sec.

Out after 42 sec.

In after 18 sec.

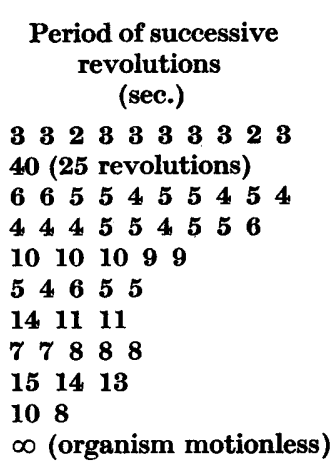

Average time in seconds for each revolution

\begin{tabular}{cc} 
Filter in & Filter out \\
2.8 & - \\
\hline 4.9 & $1 \cdot 6$ \\
$\overline{9 \cdot 8}$ & $\overline{4 \cdot 7}$ \\
$\overline{12 \cdot 0}$ & $-5 \cdot 0$ \\
- & - \\
$14 \cdot 0$ & -4 \\
- & $9 \cdot 0$ \\
- & -
\end{tabular}

Table 3. Activity of an organism exposed constantly to the radiant energy of the microscope lamp

\begin{tabular}{|c|c|}
\hline \multicolumn{2}{|c|}{$\begin{array}{l}\text { Period of exposure } \\
\text { to radiant heat } \\
\text { (hr. min.) }\end{array}$} \\
\hline 0 & 5 \\
\hline 0 & 40 \\
\hline 1 & 10 \\
\hline 2 & 10 \\
\hline $\mathbf{3}$ & 10 \\
\hline 3 & 25 \\
\hline 4 & $\mathbf{0}$ \\
\hline 5 & 0 \\
\hline
\end{tabular}

Period of exposure (hr. min.)

Period of ten consecutive revolutions
$\begin{array}{lllllllllll}\text { (sec.) } \\ 6 & 7 & 6 & 5 & 6 & 7 & 6 & 6 & 6 & 6 \\ 7 & 6 & 6 & 5 & 6 & 6 & 5 & 6 & 6 & 6 \\ 6 & 6 & 5 & 4 & 6 & 5 & 5 & 7 & 5 & 5 \\ 6 & 6 & 8 & 6 & 6 & 6 & 7 & 6 & 7 & 7 \\ 7 & 7 & 8 & 6 & 7 & 7 & 7 & 7 & 8 & 7 \\ 8 & 8 & 8 & 9 & 7 & 7 & 7 & 8 & 8 & 8 \\ 11 & 12 & 10 & 12 & 10 & 11 & 13 & 12 & 12 & 11 \\ 15 & 16 & 15 & 18 & 21 & 16 & 15 & 19 & 16 & 16\end{array}$

$\begin{gathered}\text { Average period } \\ \text { of revolution } \\ \text { (sec.) }\end{gathered}$
$\mathbf{6 \cdot 1}$
$\mathbf{5 \cdot 9}$
$\mathbf{5 \cdot 4}$
$\mathbf{6 \cdot 5}$
$\mathbf{7 \cdot 1}$
$\mathbf{7 \cdot 8}$
$\mathbf{1 1 \cdot 4}$
$\mathbf{1 6 \cdot 7}$

tions was observed at intervals, the first observation being $5 \mathrm{~min}$. after exposure to the light, when the rotation rate had become regular (Table 3).

Then the heat filter was inserted to ascertain whether, after a rest, the organism would regain its maximum speed. As soon as the filter was inserted movement ceased, and after about 10 sec. it was resumed but very slowly. After the filter had remained in for $40 \mathrm{~min}$. the rate was an average of $25.6 \mathrm{sec}$./ rev. The filter was removed after $45 \mathrm{~min}$. and the average time for the first 5 rev. was 13.8 sec., which was faster than that observed nearly an hour before; but the second 5 rev. averaged $27 \cdot 2$ sec. which was even slower than the rate when the heat filter was in. Ten minutes later, exposed to the lamp without a filter, each revolution took 28.5 sec. These observations show the gradual development of fatigue in the organism after about $3 \mathrm{hr}$.

Constancy of the rate of movement. The figures given in Table 3 show that there is a very considerable constancy in the rate of revolution at any one time. At one period the organism was timed for fifty consecutive revolutions. The times were, as usual, recorded to the nearest second.

The results were as follows: 2 rev. were made in 6 sec. each; 20 rev. were made in 7 sec. each; 21 rev. were made in 8 sec. each; 7 rev. were made in 
9 sec. each. It is clear, therefore, that the organism maintains a fairly constant rate of rotation.

Reversal of direction of movement. The insertion of a heat filter not only can immediately stop flagellar movement in slide cultures but can also induce a reversal of the direction of movement. This reversal was not seen in coiled forms and only very seldom in the very common forms, which were bent double and were travelling along with the bend foremost. It was especially noticeable in the short- or medium-length filaments which had remained more or less straight. A filament would be travelling in a certain direction across the field, say to the right. When the filter was inserted it stopped and after a second or two moved slightly to the left. If the filter remained in it would after a short delay move slowly to the left. When the filter was removed as soon as the first movement to the left was seen, the organism would dart forward in that direction. If then the filter was reinserted, the organism would stop and again reverse its direction. This to-and-fro control of the organism by manipulating the filter could be kept up for a long time. When the organism was escaping from the microscope field it could be stopped and brought back by the insertion of the filter.
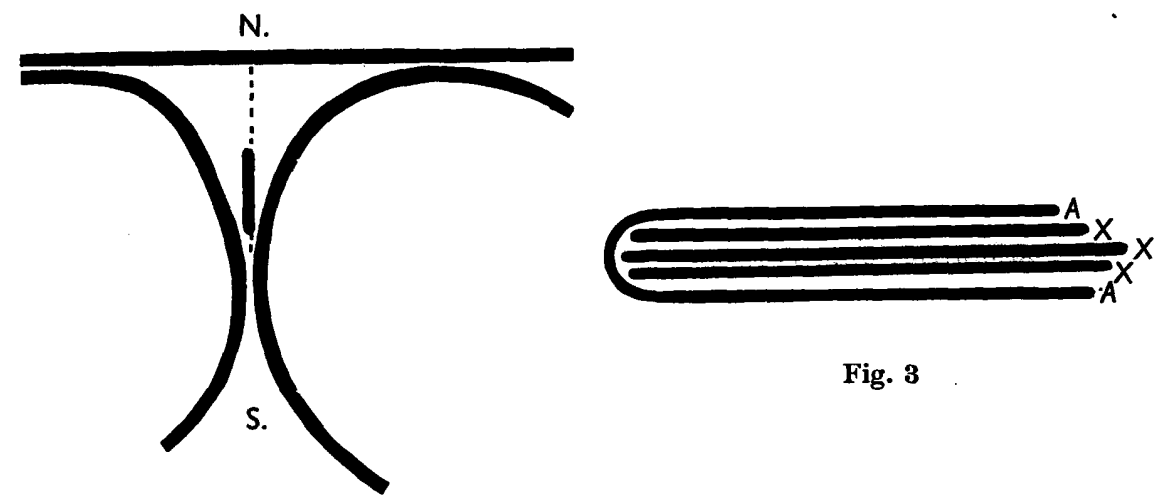

Fig. 3

Fig. 2

Fig. 2. Reversal of direction. Dotted line shows the path of the organism.

Fig. 3. Reversal of direction. Under appropriate heat stimulus the bent filament $A A$ maintains its direction of movement, while the straight filaments $X X X$ reverse their direction of movement and leave the loop.

I observed one very good example for a considerable period. A short organism only about twice as long as a normal $P r$. vulgaris was moving backwards and forwards in a north-south direction in a space only about twice as long as itself. It was enclosed between filamentous forms as shown in Fig. 2. With the heat filter in it moved gently up and down. The flagella could be seen waving gently on both sides behind the organism. The filter was removed when it had just started to move north. Almost immediately the flagella became violently active and the organism was driven against the north barrier where it remained motionless while the flagella continued in active motion. 
The filter was again inserted, the flagellar motion ceased and then it slowly returned south. Then it continued to move gently north and south while the filter was in. When it reversed its motion the flagella which had been active at one end remained stationary, but flagellar activity could be seen at the other end and the bacillus was pushed between the groups of resting flagella which appeared to fold themselves back along the body. This was repeated many times, always with the same result.

It is very common in these slide cultures to see loops of organisms moving together. In Fig. 3 there is one on the outside bent over in the middle $(A)$ and inside this there are three straight rods $(X X X)$. This loop could be emptied of its straight elements by inserting the heat filter which induced reversal of direction in the straight rods while the bent $\operatorname{rod}(A)$ continued in its original path.

The insertion of the heat filter stops flagellar movement, and it is evident that it is then that there is some impulse for the organism to reverse its direction.

\section{Movement of flagella attached to organisms which remain stationary}

So far we have been dealing with the actual movement of organisms. At the same time the flagella of these organisms could be clearly seen and, as we have stated in a previous communication (Fleming et al. 1950), flagellar movement may precede movement of the organism. In the slide cultures it is

Table 4. Duration of movement of flagella attached to twenty different immobile organisms after varying periods of rest from heat stimulation

Period that culture was screened from radiant heat before observation (sec.)

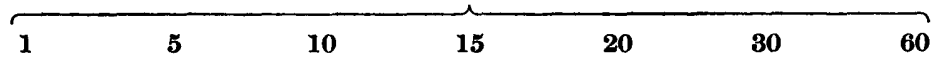

\begin{tabular}{|c|c|c|c|c|c|c|c|}
\hline Organism & & & tion & llar & lent & & \\
\hline 1 & 7 & 20 & - & 25 & - & 37 & 43 \\
\hline 2 & 6 & 14 & - & - & 一 & 31 & - \\
\hline $\mathbf{3}$ & - & 10 & 18 & - & 19 & - & $\mathbf{3 0}$ \\
\hline 4 & - & 13 & 16 & 18 & 20 & - & 18 \\
\hline 5 & 7 & 16 & 22 & - & 20 & - & 29 \\
\hline 6 & - & 17 & 23 & - & - & - & - \\
\hline$\gamma$ & - & 16 & 18 & - & 23 & - & 27 \\
\hline 8 & - & 14 & 21 & - & 25 & - & 23 \\
\hline 9 & - & - & $\mathbf{3 1}$ & - & - & $\mathbf{3 6}$ & 47 \\
\hline 10 & 15 & - & - & 37 & - & - & - \\
\hline 11 & - & 27 & 46 & - & 一 & - & 61 \\
\hline 12 & - & 14 & 22 & 34 & - & 46 & 60 \\
\hline 13 & $\div$ & 15 & 18 & 20 & 21 & 26 & 29 \\
\hline 14 & - & 15 & 17 & - & 27 & $\mathbf{3 0}$ & 37 \\
\hline 15 & - & 21 & 25 & 35 & 31 & 37 & - \\
\hline 16 & - & 22 & 29 & - & $\mathbf{3 3}$ & $\mathbf{3 6}$ & 43 \\
\hline 17 & 10 & 24 & 一 & 38 & - & 47 & 52 \\
\hline 18 & 15 & 23 & - & - & - & 47 & 54 \\
\hline 19 & 20 & 29 & $\mathbf{3 4}$ & $\mathbf{3 7}$ & $\mathbf{3 9}$ & 45 & 50 \\
\hline 20 & 12 & 19 & 23 & 23 & 25 & 28 & - \\
\hline
\end{tabular}


common to find active flagellar movement while the organism is stationary. Just like the movement of the whole organism, this flagellar movement continues for a longer time after a longer period of rest; and, further, the latent period, i.e. the time between the removal of the heat filter and the commencement of active flagellar movement, is longer with the shorter periods of rest. Many observations have confirmed these findings. Table 4 gives the actual periods of flagellar movement after various periods of rest or comparative rest when the heat filter was inserted.

Naturally with different individual organisms the times varied, but there is a very good agreement in the relative periods of movement after long and short periods of rest.

Latent period between the time the heat filter was removed and the commencement of violent flagellar movement. Extended observations have confirmed our statement that with short periods of rest the latent period was longer. I cannot go further than this. With more elaborate timing apparatus it would be possible to give accurate figures but there is no doubt that with short periods of rest, e.g. 1 or 2 sec., the latent period is much longer than with rest periods of 30-60 sec.

\section{DISCUSSION}

All the observations were made on Proteus vulgaris grown on penicillin agar; these are not normal organisms. The penicillin allows growth but inhibits cell division so all the filaments on which observations were made were compound organisms. They possessed very long flagella which wound themselves into undulating 'ropes', easily visible by phase-contrast microscopy.

There are in these cultures long filaments which move with an undulating motion exactly like a snake, but there are many others in which no motion of the body can be seen but which have active flagella which could be responsible for the movement. The flagella respond to the stimulus of very small changes in radiant heat exactly as do the motile organisms. It may be that the flagella are a type of extra-corporeal muscle which will respond to stimuli just as do muscle fibres. Heat is the only stimulus which has been investigated, but it will be interesting to try various forms of electric stimulation.

The amount of energy used in flagellar movement must be enormous, but the organisms can maintain their movements for several hours with little diminution in the rate of movement, although later the rate rapidly diminishes as if the organism was becoming exhausted.

I wish to thank Mr John L. Smith for the gift of a wire recorder which made this work easier.

\section{REFERENCES}

Fleming, A., Voureka, A., Kramer, I. R. H. \& Hughes, W. H. (1950). J. gen. Microbiol. 4, 257.

(Received 17 February 1950) 